\title{
Cytoadherence and its association with clinical disease in Plasmodium falciparum infections in Sri Lanka
}

\author{
S.L. Pathirana ${ }^{1 *}$, K. Ekanayake ${ }^{1,2}$, R.J. Howard ${ }^{3,4}$, K.N. Mendis ${ }^{1,5}$ and S.M. Handunnetti ${ }^{1,6}$ \\ ${ }^{1}$ Malaria Research Unit, Department of Parasitology, Faculty of Medicine, University of Colombo, Kynsey Road, Colombo 08. \\ 2 Avon Pharmo Chem (Pvt) Ltd., No 64 B 1/2. $2^{\text {nd }}$ Floor, Jambugasmulla Road, Nugegoda. \\ ${ }^{3}$ Department of Molecular Biology, DNAX Research Institute, Palo Alto, California, U.S.A. \\ ${ }^{4}$ Oakbio Inc., 265 Sobrante Way Suite T, Sunnyvale, California, USA. \\ ${ }^{5}$ Roll Back Malaria Initiative, World Health Organization, Geneva, Switzerland. \\ ${ }^{6}$ Institute of Biochemistry, Molecular Biology and Biotechnology, 90, Kumaratunga Munidasa Mawatha, University of Colombo, Colombo 03.
}

Revised: 10 January 2010; Accepted: 27 July 2010

\begin{abstract}
Cytoadherence properties of two categories of Plasmodium falciparum isolates from Sri Lanka were assessed using CHO-CD36 and CHO-ICAM-1 stable transfected cell lines, and the association with the clinical disease in patients, from whom the parasites were obtained was also analyzed. In the 40 uncomplicated malaria isolates (UM) studied, infected erythrocyte (IE) adherence to CHO-CD36 cells (25.8 \pm 2.1 ) was comparable to that of the 12 severe malaria (SM) isolates (35.0 \pm 6.4$)$. IE adherence to CHO-ICAM-1 cells of the SM isolates $(14.9 \pm 2.3)$ were significantly higher $(p=0.006)$ compared to that of UM isolates $(5.7 \pm 0.7)$. The percentage of isolates forming rosettes in the two clinical categories, UM and SM, were $69 \%$ and $42 \%$ respectively. However, the mean rosetting values were not statistically different $(10.5 \pm 2.9$ and $2.9 \pm 1.7)$. Expression of surface antigens was studied using assays for surface immunofluorescence and antibody mediated agglutination. All 40 UM isolates were positive for expression of surface antigens. In the UM group, the intensity of clinical symptoms, as expressed by a clinical score, was significantly and positively correlated to IE adherence to CHO-ICAM-1 $(\mathrm{r}=0.54 ; \mathrm{p}=0.003)$ but not to $\mathrm{CHO}-\mathrm{CD} 36$ cells or rosetting. The intensity of clinical symptoms was also significantly correlated with peripheral blood parasitaemia of these patients $(r=0.39$; $\mathrm{p}=0.037$ ). However, the association between adherence to ICAM-1 and intensity of clinical symptoms was independent of the peripheral blood parasitaemia of the patients (multiple regression analysis controlling for parasitaemia; $\mathrm{p}=0.016$ ). These results show that IE adherence to ICAM-1 may have implications for the intensity of clinical symptoms and the severity of infection in falciparum malaria.
\end{abstract}

Keywords: Cytoadherence, pathogenesis, Plasmodium falciparum, Sri Lanka, surface antigens.

\section{INTRODUCTION}

The mechanisms of severe manifestations in falciparum malaria and contribution of parasite cytoadherence to pathogenesis of malaria has been the focus of many studies $^{1}$. Specific molecular events involved in parasite adhesion and its interactions with venular endothelial cells are important in understanding pathogenesis and have implications for therapeutic interventions. Cytoadherence involves both host and parasite molecules. During intra-erythrocytics development, asexual stages of $P$. falciparum induce modifications in antigenic, morphological and adhesive properties of the surface of infected erythrocytes, and with these changes infected erythrocytes, acquire new functional properties such as adherence to endothelial cells and uninfected red cells to form rosettes ${ }^{2}$. Adhesion of parasites confers few survival advantages, such as providing a microaerophilic environment for their maturation, escaping clearance by the spleen or by platelets and protection from opsonization with antibodies and/or complement components $^{3,4}$. Both cytoadherence properties and rosette formation with uninfected erythrocytes and adherence to endothelium in microvasculature have been associated with severe falciparum malaria ${ }^{1}$.

P. falciparum erythrocyte membrane protein-1 (PfEMP-1) belongs to a large family of variant proteins that play a central role in host-parasite interactions ${ }^{5}$. The surface location of this variant surface antigen

*Corresponding author (sisira_pathirana@yahoo.com) 
allows for binding functions but also renders the parasite vulnerable to antibody recognition. To evade the host protective immune response, the PfEMP1 family undergoes clonal variation. The different adhesive properties of infected erythrocytes are believed to be primarily determined by specific PfEMP1 proteins that bind to CD36, intercellular adhesion molecule-1 (ICAM-1) and chondroitin sulphate A (CSA) and mediate rosetting or autoagglutination and other interactions ${ }^{5}$. To date, five rosetting receptors have been identified on red blood cells (RBCs); human serum like glycosaminoglycans (GAG)s, CD36, complement receptor 1 (CR1) and Blood group antigens $A$ and $\mathrm{B}^{1}$. Of these receptors, $\mathrm{ABO}$ blood group antigens and $\mathrm{CR} 1$ has been associated with severe disease ${ }^{6,7}$. Consistent with this, it was reported that individuals having blood group $\mathrm{O}$ are relatively resistant to severe malaria compared to individuals having other blood group types ${ }^{8}$.

Cytoadherence properties, i.e. adherence to CD36 and ICAM-1 receptors, and rosetting of Sri Lankan $P$. falciparum isolates from patients having acute uncomplicated malaria (UM) and severe malaria (SM) were investigated in this study. The expression of parasite-induced surface antigens was examined by immunofluorescence and agglutination. Infected erithocyte (IE) adherence properties of the two clinical groups were compared in order to investigate their association with severity of infection. In the uncomplicated malaria group, possible association of the intensity of clinical symptoms in patients with the cytoadherence properties of the parasite isolates was examined.

\section{METHODS AND MATERIALS}

This study was part of a research programme conducted at the Malaria Research Unit, University of Colombo, in which several different aspects of pathogenesis of falciparum malaria were investigated ${ }^{8}$ in patients with $P$. falciparum infections from Sri Lanka, where low transmission and unstable malaria conditions prevail. Forty $P$. falciparum-infected patients who had more than $0.3 \%$ peripheral ring parasitaemia at the time of diagnosis and enrolment, no anti-malarial drugs administered during the present infection and no coinfection with $P$. vivax were selected for this study (mean age 32; age range 11-65; mean days of symptoms 7). Clinical assessment of SM patients was made using accepted WHO criteria for severe manifestations ${ }^{9}$. The peripheral blood parasitaemias of these patients ranged from $0.7-11.7 \%($ mean $=4.2 \%)$. The 12 severe malaria patients were those receiving intensive care in the hospitals (mean age 29; age range 3-60; mean days of symptoms 10.5). They comprised 10 patients (most of whom had cerebral symptoms) with hepatic failure, and one with hepatic and renal failure, and one with severe anaemia. In two patients, the infections were fatal and all others recovered completely without sequelae.

At the time of the diagnosis of $P$. falciparum infection, the intensity of clinical symptoms in UM patients was evaluated using a previously validated questionnaire in which the patients' perception of the intensity of 11 symptoms was scored on a scale of $0-3$ (absent- 0 , mild-1, moderate- 2 and severe- 3$)^{10}$. In every patient a numerical clinical score ranging from 0 - 33 was arrived at, which was the summation of scores for each of the 11 symptoms. Clinical evaluation using this questionnaire was begun several weeks after the commencement of the study and therefore clinical scores were not available for every patient. Their clinical scores at the time of diagnosis ranged from $15-22($ mean $=13)$, and their peripheral blood parasitaemia from $0.3-8.6 \%$ $($ mean $=1.04 \%)$. Their clinical symptoms subsided and parasitaemia resolved completely after treatment with oral chloroquine.

$P$. falciparum ring infected blood was obtained following informed consent and prior to the antimalarial treatment, from patients who were admitted to the National Hospital Colombo and General Hospital Anuradhapura, with either uncomplicated or severe malaria, during the period from July 1994 to May 1997. Blood samples were drawn into ethylenediaminetetraacetic acid (EDTA) sodium salt (2 $\mathrm{mM}$ final concentration in blood), plasma was separated and stored frozen at $-20{ }^{\circ} \mathrm{C}$. The ring-infected cells were cryopreserved. Cryopreserved parasite isolates were thawed and cultured in vitro as described previously for 22-26 h to allow maturation of the ring stages to trophozoite/schizont stage ${ }^{2}$.

Plasma from patients was defibrinated by adding $\mathrm{CaCl}_{2}$ to convert to sera. Convalescent sera were obtained directly 10-14 days after commencement of the anti-malaria treatment. Serum samples were de-complemented for $30 \mathrm{~min}$ at $56{ }^{\circ} \mathrm{C}$ and stored at $-20{ }^{\circ} \mathrm{C}$. Normal human sera (NHS), used as negative control, were obtained from individuals of blood group $\mathrm{AB}$, who had never experienced malaria before and were residents of Colombo, which is a malaria nonendemic area. Two pools of sera were prepared, namely KG-pool and GH-pool, using sera obtained from semiimmune individuals residing in a malaria endemic area, Kataragama, Sri Lanka and from hyperimmune individuals from Ghana, respectively. 
Agglutination assays were performed as described previously $^{2}$. P. falciparum-infected cells were resuspended to a $10 \%$ haematocrit in $1: 5$ dilution of serum containing $10 \mu \mathrm{g}$ ethidium bromide $/ 1 \mathrm{~mL}$ of phosphate buffered saline (PBS). This suspension $(10 \mu \mathrm{L})$ was rotated for $45 \mathrm{~min}$ at low speed in the rotator, the content was transferred to a glass slide, covered with a cover glass and examined under bright field for agglutinates. In this quantitative assay the presence of $>3$ agglutinates with 3 or more IE was considered a positive reaction.

The surface immunofluorescence assay was performed as described previously ${ }^{11}$. Briefly $6 \mu \mathrm{L}$ of a $50 \%$ suspension of cells in PBS from the culture was mixed with $30 \mu \mathrm{L}$ of 1:5 serum dilutions in PBS, and incubated for $1 \mathrm{~h}$ at $37^{\circ} \mathrm{C}$. Cells were washed and incubated for $1 \mathrm{~h}$ with 1:50 dilution of goat anti-human fluoresceneisothiocionate-conjugated antibodies. Cells were washed and resuspended in binding medium [RPMI $1640,10 \% \mathrm{FCS}(\mathrm{pH} 7.2)]$ and $10 \mu \mathrm{L}$ of this was examined under a fluorescent microscope. Presence of 20 or more IE with surface immunofluorescence in 100 microscopic fields (corresponds to 900 infected RBCs or more) was considered as a positive reaction.

IE suspensions from the in vitro culture containing late trophozoite/schizont stages were adjusted to $5 \%$ haematocrit and rosetting was assessed as described previously ${ }^{2}$. At least 300 IE were counted, and the percentage of IE bound to more than two uninfected erythrocytes was expressed as percentage of IE in rosettes.

Cytoadherence properties of IE of $P$. falciparum isolates to endothelial cell receptors, CD36 and ICAM-1 were assessed by the microassay for adherence to CHO-CD36 and CHO-ICAM-1 cells as described previously ${ }^{12}$. These are stable (Chinese Hamster Ovary) $\mathrm{CHO}$ cell lines that express either endothelial cell receptor CD36 (CHO-CD36) or CD54 (CHO-ICAM-1)

Table 1: Association between clinical symptoms, peripheral blood parasitaemias of $P$. falciparum patients and cytoadherence properties of parasite isolates

\begin{tabular}{lll}
\hline Correlates & $\mathrm{r}$ & $\mathrm{P}$ \\
\hline Bindig to CD36 VS ICAM-1* & 0.4 & 0.01 \\
Binding to ICAM-1 vs parasitaemia+ $^{*}$ & 0.36 & 0.025 \\
Binding to CD36 vs parasitaemia ${ }^{*}$ & 0.2 & 0.23 \\
CD36 vs ICAM-1 in low rosetters* & 0.54 & 0.005 \\
CD36 vs ICAM-1 in high rosetters & 0.29 & 0.301 \\
Rosetting vs binding to CD36 & 0.02 & 0.884 \\
Rosetting vs binding to ICAM-1 & 0.12 & 0.46 \\
Rosetting vs parasitaemia+ + & 0.07 & 0.693 \\
Adherence to ICAM-1 vs clinical score & 0.54 & 0.003 \\
Adherence to CD36 vs clinical score* & 0.05 & 0.81 \\
Rosetting vs clinical score* & 0.37 & 0.052 \\
Clinical score vs parasitaemia+ + & 0.39 & 0.037 \\
Adherence to ICAM-1 vs duration of symptoms $*$ & & \\
$\quad$ in patients with primary infections* & & \\
$\quad$ in the entire group* & 0.42 & 0.026 \\
\hline
\end{tabular}

CD36 - Number of infected cells adhered to 100 target cells of CHO-CD36, $(n=40)$

ICAM-1 - Number of infected cells adhered to 100 target cells of CHO-ICAM-1

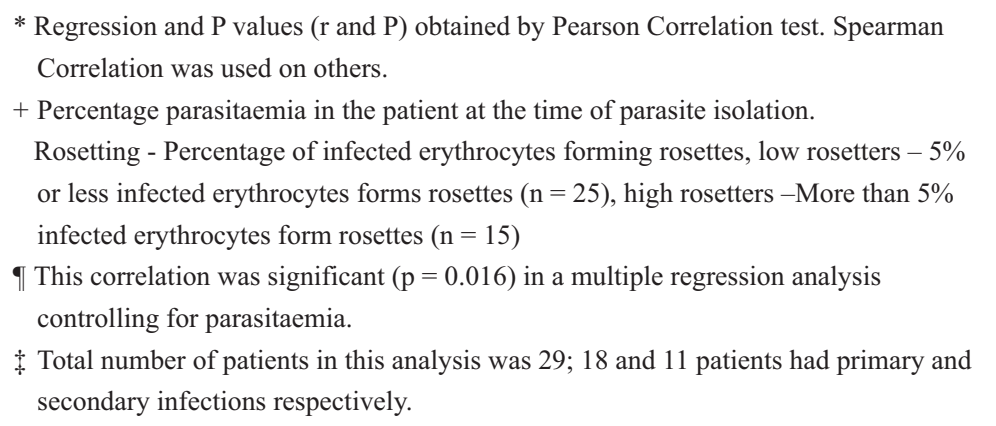


on the surface. Untransfected $\mathrm{CHO}$ cells were used as controls. One vial from a big batch of each cell type was thawed and cultured for $24 \mathrm{~h}$ in RPMI 1640, $0.2 \%$ $\mathrm{NaHCO}_{3}, 10 \%$ fetal calf serum (FCS) (pH 7.2) and used for microassay with each isolate. The microassay was performed in "parafilm" wells made in $10 \mathrm{~cm}$ tissue culture plates (FALCON 3003, USA). Each of the three $\mathrm{CHO}$ cell types were tested in triplicate wells for each isolate and adherence of four parasite isolates to these 3 target cell types was assessed in a single plate. Cell suspensions $(1 \%)$ in binding medium were incubated with $\mathrm{CHO}$ cells for $1 \mathrm{~h}$ at $37^{\circ} \mathrm{C}$. Plates were gently and carefully washed 6-8 times with binding medium until there were no settled, infected or uninfected erythrocytes in the background. The binding of trophozoite/schizontinfected cells was counted with a light microscope under oil immersion. Duplicate counts were made from each of the triplicate wells having a particular target cell type. Thus adherence to a total of 600 target cells were counted and adherence of IE was expressed as the average number of IE bound/100 target cells.

In the $\mathrm{CHO}$ cell adherence assay, binding to untransfected $\mathrm{CHO}$ cells was very low, indicating a low non-specific binding as previously shown ${ }^{12}$. The adherence to both CHO-CD36 and CHO-ICAM-1 cells in P. falciparum isolates had a strong correlation with percentage parasitemia in in vitro culture $(\mathrm{r}=0.76$;

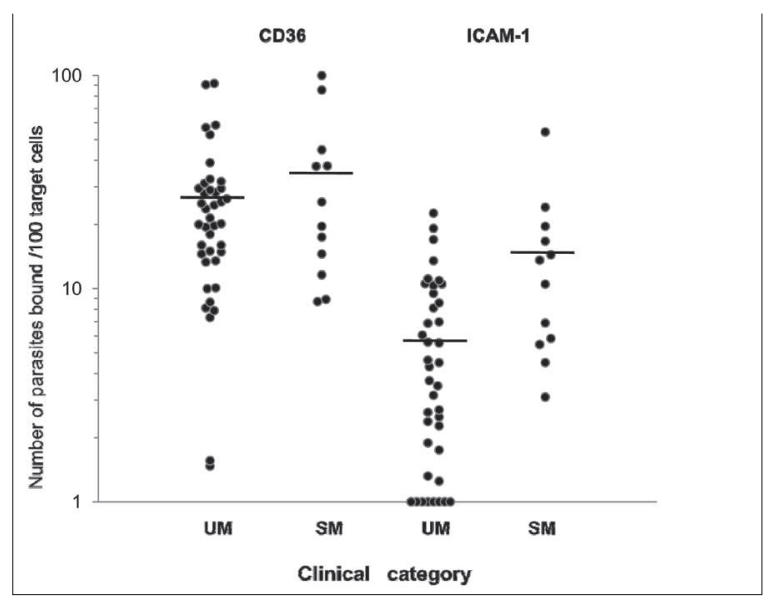

Figure 1: Intrinsic adherence of natural P. falciparum isolates to endothelial receptors, CD36 and ICAM-1 expressed on transfected CHO cells. UM - parasite isolates from uncomplicated malaria cases; SM - parasite isolates from severe malaria cases. Horizontal bar represents the mean value. $\mathrm{p}=0.0001$ and $\mathrm{r}=0.53 ; \mathrm{p}=0.0001$ respectively). Thus, the intrinsic adherence of these parasite isolates at $1 \%$ parasitaemia was compared, which was obtained by dividing the absolute adherence values by the parasitaemia of the in vitro culture.

\section{RESULTS}

The intrinsic adherence of 40 uncomplicated P. falciparum isolates to CHO-CD36 and CHO-ICAM-1 cells ranged from $1.4-91.8(25.8 \pm 2.1 ;$ mean \pm SEM $)$ and 0-22.6 (5.7 $\pm 0.7 ;$ mean \pm SEM) infected erythrocytes per 100 target cells respectively (Figure 1). Thus, the degree of adherence to CHO-ICAM-1 cells was lower than that to CHO-CD36 cells. The level of adherence to CD36 and ICAM-1 observed in these natural isolates in Sri Lankan patients was comparable to those reported in studies from other geographical areas ${ }^{2,13}$. Further, there was a significant positive correlation between the adherence of CD36 and ICAM- $1(\mathrm{r}=0.40 ; \mathrm{p}=0.01)$ (Table 1). Interestingly, this correlation applied only to, and was very strong in the low rosetting isolates $(\mathrm{r}=0.54$; $\mathrm{p}=0.005$ ), while in the high rosetting isolates there was no significant correlation between binding to $\mathrm{CHO}-$ CD36 and CHO-ICAM-1 cells (Table 1). IE adherence to CHO-ICAM-1 cells was positively correlated with the percentage parasitaemia in the peripheral blood of the patient from whom the parasites were isolated $(\mathrm{r}=0.36$; $\mathrm{p}=0.025$ ).

IE adherence to CHO-ICAM-1 cells ranged 3.1 $54.3(14.9 \pm 2.3$; mean $\pm \mathrm{SEM})$ in parasite isolates from SM patients and was significantly higher than that of the uncomplicated patients $(\mathrm{p}=0.006, \mathrm{z}=2.74)$ (Figure 1). However, IE adherence to $\mathrm{CHO}-\mathrm{CD} 36$ cells in SM isolates ranged $8.7-108.3(35.0 \pm 6.4$; mean $\pm \mathrm{SEM})$ did not differ from those of the UM isolates (Figure 1).

In $29 \%$ of the parasite isolates a higher percentage of the IE ( $>5 \%)$ formed rosettes. In a majority $(40 \%)$ of isolates $1-5 \%$ of IE formed rosettes and the rest $(31 \%)$ showed no evidence of rosetting at all (Figure 2). The lack of rosetting in these isolates is likely to have been a true negative phenotypic expression (rather than poor parasite growth in in vitro culture or other such technical reasons) because all these non-rosetting isolates showed adherence to both CHO-CD36 and CHO-ICAM-1 cells. Rosetting of IE from the SM patients $(\mathrm{n}=12)$ ranged from $0-20 \%$, but $58 \%$ of the SM isolates were nonrosetters. Rosetting of SM isolates $(2.9 \pm 1.7$; mean \pm SEM) was not significantly different from that of UM patients $(10.5 \pm 2.9$; mean \pm SEM $)$. All $40 \mathrm{UM}$ isolates agglutinated with the two pools of immune sera (KG and $\mathrm{GH})$. Seventy two and $88 \%$ of the isolates were positive 


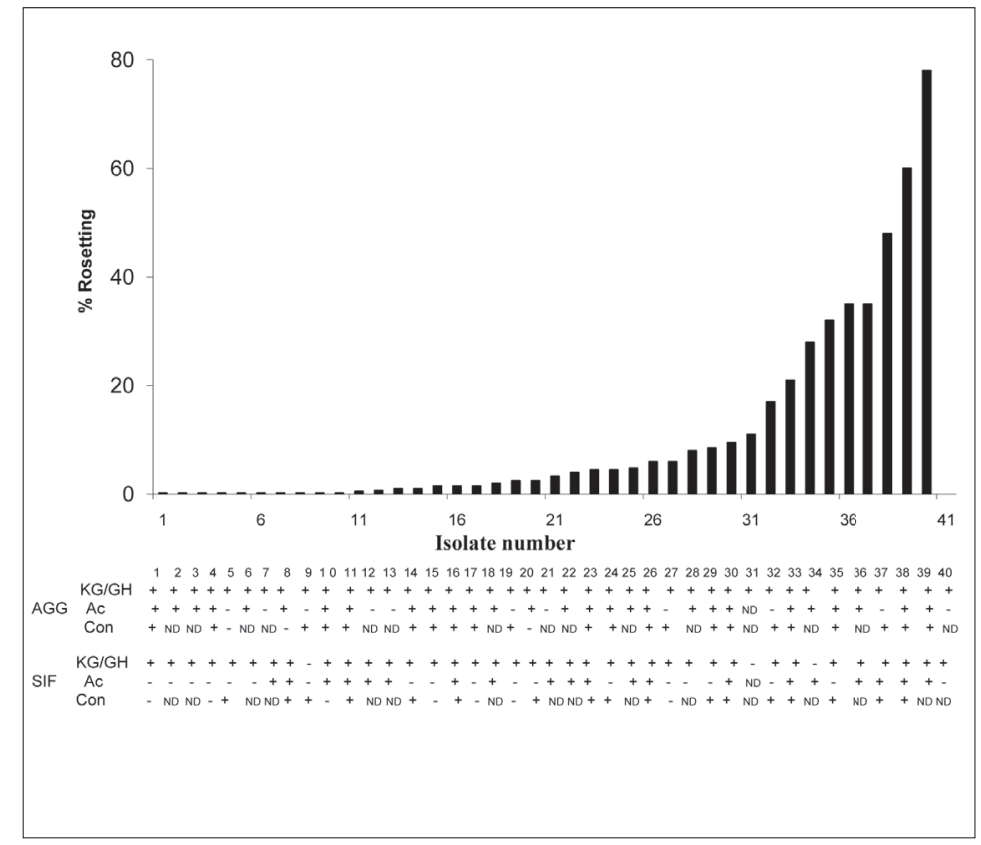

Figure 2: Percentage rosette formation of UM parasite isolates and their reactivity in antibody-mediated agglutination and surface immunofluorescence assay. AGG -agglutination; SIF - surface immunofluorescence; Ac, Con \& KG/GH are reactivity with acute sera, convalescent sera and Kataragama or Ghanian serum pools respectively; ND - not done.

with the autologous acute and convalescent sera (Figure 2). In surface immunofluorescence, $93 \%$ of the isolates gave positive results with two pools of sera. Fifty one and $71 \%$ of isolates reacted positively with autologous acute and convalescent sera respectively (Figure 2). Thus, all the parasite isolates used in the study expressed surface antigens as detected by either the aggluatination or surface immunofluorescence or functional assays, i.e. assay for CD36 and ICAM-1 adherence and rosette formation.

In UM isolates, adherence to ICAM-1 was significantly and positively correlated with the intensity of clinical symptoms (total clinical score) in the patient from whom the isolate was obtained $(\mathrm{r}=0.54 ; \mathrm{p}=0.003)$ (Table 1). No such correlation was observed between the clinical score and either the adherence to CD36 or rosetting (Table 1). The intensity of clinical symptoms was also significantly correlated with peripheral blood parasitaemia of these patients $(\mathrm{r}=0.39 ; \mathrm{p}=0.037)$. Multiple regression analysis controlling for parasitaemia showed that the association between IE adherence to ICAM-1 and the intensity of clinical symptoms was independent of the parasitaemia $(\mathrm{p}=0.016)$. This data suggests that
IE adherence to ICAM-1 may be causatively related or that it is a marker for severe clinical disease in malaria. Further, there was a positive correlation between IE adherence to ICAM-1 and the duration of symptoms, in patients having primary infections (first infection in their life) $(\mathrm{r}=0.42 ; \mathrm{p}=0.026 ; \mathrm{n}=18)$ and a positive trend was observed within the entire UM group $(\mathrm{r}=0.31$; $\mathrm{p}=0.05$ ). This suggests that ICAM- 1 expression may be upregulated or higher-affinity binding to ICAM-1 may occur during infections in "malaria naïve" individuals.

\section{DISCUSSION}

This study was focused on cytoadherence properties of Sri Lankan P. falciparum isolates and of the three cytoadherence properties tested, a significant association was observed between cytoadherence to ICAM-1 and the clinical disease. In the uncomplicated infections, a strong positive correlation was found between the binding of IE to ICAM-1 and the intensity of symptoms which was independent of the parasitaemia. These findings suggest that the two properties of the parasite isolates, achieving high rates of multiplication in blood, and high binding to ICAM-1, are independent virulent 
properties. Furthermore, parasite isolates obtained from patients with severe malaria had significantly higher levels of binding to ICAM-1 than those obtained from uncomplicated infections, implicating this adherence property in the pathogenesis of severe malaria, a finding which is consistent with a previous study ${ }^{14}$.

Virulence properties described previously include high rosette formation of parasite isolates from cerebral and severe malaria patients ${ }^{6,15}$ and other properties such as the ability to induce high TNF-alpha levels ${ }^{15}$ and other cytokines such as IL-10 ${ }^{16}$. A study conducted in Kenya with a larger sample of falciparum isolates had shown that binding to ICAM-1 in the cerebral and nonsevere groups was significantly higher than that in the asymptomatic controls implicating binding to ICAM-1 in clinical illness ${ }^{14}$.

The presence of very few isolates with high binding to ICAM-1 among natural parasite isolates appears to suggest that only a small proportion of infections give rise to severe forms of the disease. The overlapping range of IE adherence to ICAM-1 observed in isolates from the UM and SM patients in this study raises the question as to whether the high ICAM-1 binding parasites are potentially "virulent strains" which were being diagnosed and treated early, thus preventing possible severe manifestations in vivo as suggested by Gupta and others ${ }^{17}$. It is very likely that the disease outcome of an infection depends on the intrinsic parasite properties as well as on several other determinants such as predisposing host genotypes that code for specific alleles for promoting the expression of TNF-alpha, ABO blood group antigens, ICAM-1 ${ }^{\text {kilifi }}$ mutation, ICAM-1 rs5498 (exon 6) G allele and CD36 exon 1a A allele, CR1 polymorphisms, and indeed the strength of the prevailing health system to deliver early and effective treatment ${ }^{7,8,18,19}$. Depending on the host TNF allele type, in patients having TNF $\alpha * 1, * 2$ genotype, high levels of TNF can be induced, which can in turn upregulate the ICAM-1 expression on endothelial cells and thus, select for high ICAM-1 binders; which could be a causative factor for development of severe symptoms. This is supported by the findings of the present study, that there is a tendency to have high IE adherence to ICAM-1 in uncomplicated patients in whom the duration of symptoms is longer. If the diagnosis and treatment is delayed, this may potentially contribute to severe pathology, possibly through selecting for a high ICAM-1 binding parasite population that may cause increased adherence. In fact, among the patients with their very first infections, there was a significant correlation between the duration of symptoms and binding to ICAM-1 implying a tendency for an increase in intrinsic binding to ICAM-1 with time during acute infections.
Inclusion of parasites from asymptomatic patients would have clarified whether the increased binding to ICAM-1 and CD36 is actually associated with clinical severity. However, binding of asymptomatic cases could not be performed since parasitaemia of all asymptomatic cases encountered were well below the cut off level of parasitaemia used in this study.

Most isolates that gave strong binding to ICAM-1 were also high $\mathrm{CD} 36$ binders indicating that there was co-expression of high affinity binding domains to both receptors. This is consistent with the previous report that had implicated the co-expression of high affinity binding to both CD36 and ICAM-1, which could confer a functional advantage on the parasite resulting in increased cytoadherence ${ }^{20}$. This is supported by the fact that PfEMP1, the variant surface antigen of $P$. falciparuminfected red cells, contain the binding domains for both CD36 and ICAM- ${ }^{5}$.

Interestingly, the correlation between parasite adherence to CHO-CD36 and CHO-ICAM-1 cells was seen only in the low rosetting isolates and was absent in high rosetters. This lack of correlation may be due to rosette formation in the in vitro cultures, which would physically interfere with parasite adherence to CD36 and ICAM-1 expressed on CHO cells. Other than the steric hindrance from uninfected erythrocytes in rosettes, there could be competition for receptors on infected erythrocytes. It is, however, quite possible that a correlation exists between parasite adherence to $\mathrm{CHO}-$ CD36 and CHO-ICAM-1 cells even in the high rosetting isolates, which is not detectable by our in vitro binding assay. Apart from this, since two different lines of CHO cells that express either CD36 or ICAM-1 were used, it was possible to compare the intrinsic adherence to one endothelial receptor independent of the other, unlike in assays used in previous studies where C32 melanoma cells or human umbilical vein endothelial cells (HUVEC) were used as target cells, which express more than one endothelial receptor. The adherence to endothelial cells in vivo may depend on several factors which are very difficult to be reproduced and analysed i.e., i) expression of multiple endothelial receptors on target cells and the efficiency of adhesion may depend on the infected cell interaction with these different receptors, ii) differential expression of each of these receptors during an acute infection and iii) functions of each receptor under shear stress conditions which prevail under physiological conditions. It is evident from this study, that binding to ICAM-1 is associated with clinical outcome in falciparum 
malaria at two levels of clinical intensity as discussed above. These results merit pursuing studies to further elucidate the role of binding to ICAM-1 in pathogenesis of malaria.

\section{Acknowledgement}

The authors gratefully acknowledge co-operation extended by the patients and staff of the National Hospital of Sri Lanka and the General Hospital, Anuradhapura, Dr Harsha Alles and Sumith Bandara for clinical investigations of patients, Prof A.R. Wickremasinghe for valuable advice given for statistical analysis, K.L.R.L. Perera, U. Wijesinghe, J. Rajakaruna, S. Weerasinghe, M. Kularathna and G.M.G. Kapilananda for technical assistance, and Ms T. Jayasekera for secreterial assistance. This investigation received financial support from the UNDP/World Bank/WHO Special Programme for Research and Training in Tropical Diseases (TDR) grant ID 900683 and 940434.

\section{References}

1. Chen Q., Schlichtherle M. \& Wahlgren M. (2000). Molecular aspects of severe malaria. Clinical Microbiology Reviews 13(3): 439-450.

2. Hasler T., Handunnetti S.M., Aguiar J.C., van Schravendijk M-R., Greenwood B.M., Lallinger G., Cegielski P. \& Howard R.J. (1990). In vitro rosetting, cytoadherence, and microagglutination properties of Plasmodium falciparum-infected erythrocytes from Gambian and Tanzanian patients. Blood 76(9): 1845-1852.

3. Buffet P.A., Milon G., Brousse V., Correas J.M., Dousset B., Couvelard A., Kianmanesh R., Farges O., Sauvanet A., Paye F., Ungeheuer M.N., Ottone C., Khun H., Flette L., Guigon G., Huerre M., Mercereau-Puijalon O. \& David P. H. (2006). Ex vivo perfusion of human spleens maintains clearing and processing functions. Blood 107(9): 37453752 .

4. McMorran B.J., Marshall V.M., De. Graaf C., Drysdale K.E., Shabbar M., Smyth G.K., Corbin J.E., Alexander W.S. \& Foote S.J. (2009). Platelets kill intraerythrocytic malarial parasites and mediate survival to infection. Science 323(5915): 797-800.

5. Smith J.D., Gamain B., Baruch D.I. \& Kyes S. (2001). Decoding the language of var genes and Plasmodium falciparum sequestration. Trends in Parasitology 17(11): 538-545.

6. Rowe A., Obeiro J., Newbold C.I. \& Marsh K. (1995). Plasmodium falciparum rosetting is associated with malaria severity in Kenya. Infection and Immunity 63(6): 2323-2326.

7. Cockburn I.A., Mackinnon M.J., O’Donnel A., Allen S.J., Moulds J.M., Baisor M Bockarie M., Reeder J.C. \& Rowe J.A. (2004). A human complement receptor
1 polymorphism that reduces Plasmodium falciparum rosetting confers protection against severe malaria. Proceedings of the National Academy of Science, U.S.A. 101(1): 272-277.

8. Pathirana P.P.S.L., Alles H.K., Perera M.K., Bandara S., Phone-Kyaw M., Mendis K.N. \& Handunnetti S.M. (2005). ABO blood group types and protection against severe Plasmodium falciparum malaria. Annals of Tropical Medicine and Parasitology 99(2): 119-124.

9. Warrell D.A., Molyneux M.E. \& Beales P.F. (1990). Severe and complicated malaria. Transactions of the Royal Society of Tropical Medicine \& Hygiene 84(2): 1-65.

10. Karunaweera N.D., Carter R., Grau G.E. \& Mendis K.N. (1998). Demonstration of anti-disease immunity to Plasmodium vivax malaria in Sri Lanka using a quantitative method to assess clinical disease. American Journal of Tropical Medicine \& Hygiene 58(2): 204-210.

11. Handunnetti S.M., Mendis K.N. \& David P.H. (1987). Antigenic variation of cloned Plasmodium fragile in its natural host Macaca sinica: sequential appearance of successive variant antigenic types. Journal of Experimental Medicine 165(5): 1269-1283.

12. Hasler T., Albrecht G.R., van Schravendijk M-R., Aguiar J.C., Morehead K.E., Pasloske B.L., Ma C., Barnwell J. W., Greenwood B. \& Howard R.J. (1993). An improved microassay for Plasmodium falciparum cytoadherence using stable transformants of Chinese hamster ovary cells expressing CD36 or intercellular adhesion molecule-1. American Journal of Tropical Medicine \& Hygiene 48(3): 332-347.

13. Ho M., Singh B., Looareesuwan S., Davis T.M.E., Bunnag D. \& White N.J. (1991). Clinical correlates of in vitro Plasmodium falciparum cytoadherence. Infection and Immunity 59(3): 873-878.

14. Newbold C.I., Warn P., Black G., Berendt A., Craig A., Snow B., Msobo M., Peshu N. \& Marsh K. (1997). Receptor-specific adhesion and clinical disease in Plasmodium falciparum. American Journal of Tropical Medicine \& Hygiene 57(4): 389-398.

15. Ringwald P., Peyron F., Lepers J.P., Rabarison P., Rakotomalala C., Razanamparany M., Rabodonirina M., Roux J. \& Le Bras J. (1993). Parasite virulence factors during falciparum malaria: rosetting, cytoadherence and modulation of cytoadherence by cytokines. Infection and Immunity 61(12): 5198-5204.

16. Baptista J.L., Wery M. \& van der Stuyft P. (1997). Influence of storage temperature on estimates of tumour necrosis factor in plasma samples from patients with cerebral malaria. Annals of Tropical Medicine and Parasitology 91(4): 429-431.

17. Gupta S., Hill A.V.S., Kwiatkowski D., Greenwood A.M., Greenwood B.M. \& Day K.P. (1994). Parasite virulence and disease patterns in Plasmodium falciparum malaria. Proceedings of the National Academy of Science USA 91(9): 3715-3719.

18. Craig A. \& Scherf A. (2000). Transcriptional analysis of Plasmodium falciparum. Trends in Microbiology 8(8): 350-351. 
19. Singhe S., Qidwai T., Kanchan K., Anand P., Jha G.N., Pati S.S., Mohanty S., Mishra S.K., Tyagi P.K., Sharma S. K., Venkatesh V. \& Habib S. (2008). Variation in host genes encoding adhesion molecules and susceptibility to malaria in India. Malaria Journal 7(12):250.
20. Chaiyaroj S.C., Coppel R.L., Novakovic S. \& Brown G.V. (1994). Multiple ligands for cytoadherence can be present simultaneously on the surface of Plasmodium falciparum-infected erythrocytes. Proceedings of the National Academy of Science USA 91(23):10805-10808. 